\title{
Don't slip through the net
}

\section{R Al-Shahi, C Kennard}

\section{JNNP could help you become a technophile}

t is possible to thrive in the modern world without recourse to the internet. But only just. If you are a "technophobe", deterred by the daunting size and complexity of this continually evolving medium, JNNP could help you become a "technophile".

Fewer than four decades ago, the cold war motivated the creation of the internet. Academic inspiration subsequently harnessed the internet's potential in its two best-known components: email and the world wide web. Born of a need for communication and maturing through a hunger for information, these two human desires have made the internet indispensable.

The overwhelming size of the internet makes it a formidable repository of knowledge. The figure below shows the Internet Software Consortium's latest internet domain survey (www.isc.org), which estimates that there are $>160$ million "hosts" and that their greatest proliferation has occurred in the last four years.

It is, of course, impossible to know how many people use the internet, but an educated guess is that $\sim 580$ million

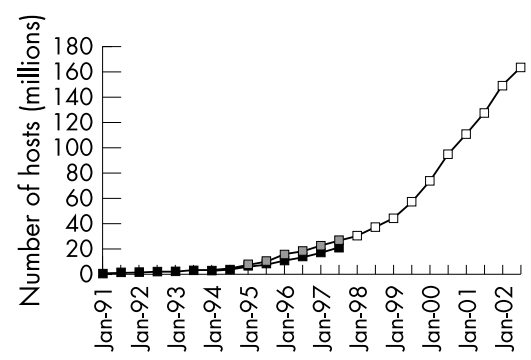

Figure 1 Internet domain survey host count. Reproduced with permission. people across the world (almost 10\% of the global population) were online in May 2002 (www.nua.com). Health is probably the largest single category of information hosted by the world wide web, and over two thirds of the online public-known as "cyberchondriacs" seek information about it. ${ }^{1}$

Despite its impact, the internet is still flawed. Access in the developing world remains limited, but initiatives like Digital Island seek to broaden the availability of material-such as JNNP-and make the world a smaller place. ${ }^{2}$ While freedom to publish is part of the essence of the world wide web, cultural constraints will preclude consensus about what constitutes appropriate censorship. ${ }^{3}$ The notorious excess of poor quality informationlargely due to lack of regulation and commercial exploitation-has become enough of a priority for the $B M J$ to devote its 9 March 2002 (Evaluating the quality of health information on the internet) theme issue to it. A recent survey is cause for some optimism; almost half of the "cyberchondriacs" in the USA and France were found to visit medical journals and academic or research institutions, closely followed by commercial health web sites. ${ }^{1}$ Furthermore, the entire contents of two online evidence based health resources (Clinical Evidence and the Cochrane Library) were made available to the public in September 2002 through the National Electronic Library for Health (www.nelh.nhs.uk).

So how can JNNP help you cope with this immortal yet imperfect (but improving) medium?

Since 15 March 1999 the full text of the Journal has been available at www.jnnp.com, ${ }^{4}$ as have customised @lerts, collected resources, and emailed tables of contents, with free back issues, rapid responses, and electronic manuscript submission via Bench $>$ Press introduced recently. In this issue, the first of a series of review articles deals with the internet itself. ${ }^{5}$ Subsequent articles will focus on resources for neurology (including neurophysiology and neuroradiology), neurosurgery (including neuropathology), and psychiatry (including neuropsychiatry). Every review will be free at www.jnnp.com, and the recommended web sites are available as downloadable Bookmarks/Favorites at http://jnnp.bmjjournals.com/misc/ bookmarks.shtml. If you are already a "technophile", or if we succeed in converting you, join us in our attempt to keep pace with the internet in the monthly Neuronline filler section. ${ }^{6}$

J Neurol Neurosurg Psychiatry 2002;73:611

\section{Authors' affiliations}

R Al-Shahi, Web Editor, JNNP, Department of Clinical Neurosciences, Western General Hospital, Crewe Road, Edinburgh EH4 2XU, UK; rustam.al-shahi@ed.ac.uk

C Kennard, Editor, JNNP, Division of Neuroscience and Psychological Medicine, Imperial College School of Medicine, Charing Cross Hospital, Fulham Place Road, London W6 8RF, UK; c.kennard@ic.ac.uk

\section{REFERENCES}

1 Four-nation survey shows widespread but different levels of Internet use for health purposes. Health Care News 2002;2:1-4. (www.harrisinteractive.com)

2 Kennard C. Getting our Journal to developing countries. J Neurol Neurosurg Psychiatry 2001;71:711.

3 Gittings J. Google is back in China but don't try asking any difficult questions. The Guardian 14 September 2003: 3.

4 Kennard C. Same name, new face for JNNP. www.jnnp.com. J Neurol Neurosurg Psychiatry 1999:66:415.

5 Al-Shahi R, Sadler M, Rees G, et al. The internet. J Neurol Neurosurg Psychiatry 2002;73:619-628

6 Macleod M. Neuronline: PubMed: http://www.pubmed.org. J Neurol Neurosurg Psychiatry 2002;73:764. 


\section{Fetal striatal transplantation in Huntington's disease: time for a pause}

\section{R L Albin}

The need to look at the rationale for fetal striatal transplantation

68 $\mathrm{n}$ this issue of the Journal (pp 678685), ${ }^{1}$ the NEST-UK consortium reports safety data on four subjects with Huntington's disease receiving unilateral intrastriatal transplantation of human fetal striatal tissue. This well designed and carefully executed study adds to the small amount of previous data on fetal striatal grafting for Huntington's disease. ${ }^{234}$ Groups in California and France reported a total of eight subjects receiving bilateral engraftment without complications and either no change in clinical outcome measures or some improvement. ${ }^{23}$ In another series of seven patients undergoing bilateral engraftment, there were four subdural haematomas (in three subjects), two requiring neurosurgical drainage. ${ }^{4}$ These latter subjects may have had more advanced Huntington's disease with a greater degree of brain atrophy, and this may be a contraindication to engraftment. The NEST-UK investigators plan a second phase safety study examining bilateral engraftment with a total of 10 subjects (including the four in the present report), and a two year follow up to generate preliminary efficacy data. This systematic approach is commendable.

The accumulated data, however, raise real questions about moving forward into efficacy trials. An incidence of two symptomatic subdural haematomas among 19 subjects is unacceptable. It is imperative that efficacy trials be controlled appropriately. The recent experience with fetal tissue engraftment for Parkinson disease revealed the existence of large magnitude and remarkably long duration placebo effects. Some form of sham surgery control is necessary. The NEST-UK protocol involves general anaesthesia, the surgery, a week of postoperative hospita care, and immunosuppression with frequent blood monitoring. Designing an adequate and safe sham surgery protocol will be a challenge.

The rationale for the fetal striatal transplantation is questionable. Successful striatal engraftment is likely to have limited effects. The hallmark of Huntington's disease is striatal degeneration, but the disorder affects the whole brain There is significant whole brain atrophy, and neurodegeneration of the neocortex, hippocampal formation, thalamus, substantia nigra, and cerebellum have all been documented. Fetal striatal transplantation has shown efficacy in animal models of Huntington's disease, but these models use mainly toxin destruction of the striatum and do not mimic the systemic nature of Huntington's disease. If there were no alternative approaches, continued pursuit of engraftment would be rational. However, identification of the genetic defect in
Huntington's disease-expanded CAG repeats within the huntingtin locusresulted in an explosion of research on the pathogenesis of the disease. Recent advances include the development of several murine genetic models and the identification of various molecular processes as potential therapeutic targets. I believe the likelihood of an effective treatment emerging from these lines of investigation is considerably higher than the likelihood of marked improvement with fetal striatal engraftment.

Given the practical and theoretical objections to fetal striatal engraftment for Huntington's disease, the best approach would be for the NEST-UK investigators and other groups in this area to complete their safety trials and then pause.

J Neurol Neurosurg Psychiatry 2002;73:612

\section{Authors' affiliations}

R L Albin, Department of Neurology, University of Michigan, and Geriatrics Research Education, and Clinical Center, Ann Arbor VAMC, room 4412D, Kresge III Building, 200 Zina Pitcher Place, Ann Arbor, Ml 48109-0585, USA

Correspondence to: Dr R Albin: ralbin@umich.edu

\section{REFERENCES}

1 Rosser AE, Barker RA, Harrower T, et al. Unilateral transplantation of human primary fetal tissue in four patients with Huntington's disease: NEST-UK safety report ISRCTN no 36485475. J Neurol Neurosurg Psychiatry 2002:73:678-685.

2 Kopyov OV, Jacques S, Lieberman A, et al. Safety of intrastriatal neurotransplantation for Huntington's disease patients. Exp Neurol 1998;149:97-108

3 Bachoud-Levi AC, Remy P, Nguyen JP, et al Motor and cognitive improvements in patients with Huntington's disease after transplantation. Lancet 2000;356:1975-9.

4 Hauser RA, Furtado $S$, Cimino CR, et al. Bilateral human fetal striatal transplantation in Huntington's disease. Neurology 2002;58:687-94.

\section{Neuroimaging for epilepsy: quality and not just quantity is important}

\section{J S Duncan}

\section{Current resources for neuroimaging could be used more efficiently}

$\mathrm{T}$ he value of magnetic resonance imaging (MRI) of the brain in people developing epilepsy has been amply demonstrated. ${ }^{1}$ This is particularly the case for those with medically refractory partial seizures, for whom appropriate surgical treatment may result in complete resolution of seizures in $70 \%$.
MRI scanning has become much more available over the last decade in many community hospitals, to the benefit of the populations they serve. There are some concerns, however, about quality control, and particularly because of the shortage of trained neuroradiologists. This important issue is addressed in the paper by Von Oertzen et al (this issue pp 643-647). ${ }^{2}$

It was found that MRI scans carried out and reported on in German community hospitals had a low rate of detection of focal lesions such as hippocampal sclerosis, tumours, cortical malformations, and vascular anomalies. There was a modest increase in detection rate by having these scans reviewed by expert neuroradiologists at the Bonn Epilepsy 
Centre. There was a greater increase in detection by obtaining epilepsy dedicated MRI in the centre, and focal lesions were identified in $85 \%$ of patients with unremarkable standard MRI.

Does this mean that all patients with refractory epilepsy need to be referred to specialist centres for their MRI scans? At present this would be an appropriate part of the evaluation for possible surgical treatment of the epilepsy, but it is also important to improve the diagnostic accuracy of MRI scans obtained at smaller units. There is some impact from improved scanning hardware, but the greater advance is possible from the use of MRI acquisition protocols for patients with epilepsy that may be used by radiographers without extensive specialist training. Protocols have been formulated in guidelines by the International League against Epilepsy ${ }^{3}$ that have also considered the complementary role of functional imaging with MRI..$^{5}$ A typical basic epilepsy MRI protocol would comprise a Tl weighted volume acquisition which may be reformatted in any orientation and used for volumetric measurements: proton density, T2 weighted, and fluid attenuated inversion recovery (FLAIR) acquisitions obtained with contiguous slices that cover the entire brain with as thin slices as possible and orientated perpendicular to the hippocampus. The oblique coronal orientation minimises partial volume effects that otherwise commonly obscure hippocampal sclerosis and small lesions in the temporal lobe, and also displays the rest of the brain in a clear and logical fashion. Standardisation of acquisition protocols also facilitates subsequent review of images at a specialist centre and may avert the need for repeat scans.

There is a clear need for an increased number of trained neuroradiologists to direct image acquisition and to report on MRI scans. In the meantime, current resources could be used more efficiently by the increased use of standardised acquisition protocols and effective telereporting in the managed clinical networks of community hospitals and regional specialist centres.
J Neurol Neurosurg Psychiatry 2002;73:612-613

\section{Author's affiliations}

J S Duncan, Department of Clinical and Experimental Epilepsy, National Hospital for Neurology and Neurosurgery, Queen Square, London WCIN 3BG

Correspondence to: Professor J S Duncan; i.duncan@ion.ucl.ac.uk

\section{REFERENCES}

1 Duncan JS. Imaging and epilepsy. Brain 1997; 120:339-78.

2 von Oertzen J, Urbach $H$, Jungbluth S, et al. Standard magnetic resonance imaging is inadequate for patients with refractory focal epilepsy. J Neurol Neurosurg Psychiatry 2002;73:643-647.

3 Barkovich AJ, Berkovic SF, Cascino GD, et al. Recommendations for neuroimaging of patients with epilepsy. Epilepsia 1997;38: 1255-6.

4 Berkovic SF, Duncan JS, Barkovich A, et al. ILAE neuroimaging commission recommendations for neuroimaging of persons with refractory epilepsy. Epilepsia 1998;39:1375-6.

5 Duncan JS, Ali R, Barkovich J, et al. Neuroimaging subcommission of ILAE. Recommendations for functional neuroimaging of persons with epilepsy. Epilepsia 2000;41:1350-6.

\section{CJD: are there distinct neuropsychological features?}

\section{R Knight}

\section{Different faces wearing the same expression: is there a core neuropsychological deficit in sporadic CJD?}

A $s$ with many diseases originally defined on purely clinicopathological phenomenological grounds, "Creutzfeldt-Jakob disease" (CJD) has been subject to various "lumping" and "splitting" processes over the years. ${ }^{1}$ The demonstration of laboratory transmissibility and the central role of the prion protein have served to unify the concept of CJD. Sporadic CJD (sCJD) remains, of course, defined by the absence of a known cause, in contradistinction to genetic, iatrogenic, and variant CJD. Despite this background unification, there is notable clinical phenotypic variation in SCJD including age of onset, duration of illness, and presenting symptom(s) in particular. Research has attempted to correlate these variations with molecular factors such as the PRNP genotype and the prion protein structure. ${ }^{2}$ If SCJD is indeed a "spontaneous" disease due to a chance protein structural change or somatic PRNP mutation, then the different presentations may simply reflect the usual function of the initially affected part of the CNS. Whatever the explanation, the current view of SCJD is of an illness that may present in a variety of ways, including cerebellar ataxia, cortical blindness, dysphasia, and other deficits but which rapidly evolves into a global dementia with features of widespread brain disease. While attention has been given to the different modes of "physical" neurological presentation, there has been little comment on the pattern of cognitive dysfunction. A paper by Snowden et al (this issue pp 686-694) describes some detailed observations on five cases of SCJD (and one presumed familial case). ${ }^{3}$ The authors claim to have found a common, distinct neuropsychological profile despite different neurological presentations.

There are obvious reasons for the previous neglect of detailed cognitive assessments, as the authors indicate, including the rarity of SCJD and its rapid clinical progression (mean duration: five months). Severe neurological impairment, even mutism, may be present at first neurological assessment. ${ }^{1}$ It is therefore particularly notable that the authors were able to assess their cases, using neuropsychological tools previously developed for other dementias. Two particular findings, namely fluctuations in ability over time and variability of responsiveness will resonate with ordinary clinical observations of cases of sCJD and accord well with comments made by many relatives.

As the authors admit, these results are derived from only a small number of slightly atypical cases. One case was relatively young, four cases had longer than average durations and at least two were of unusual PRNP genotype. Further studies are therefore needed to confirm these preliminary findings.

Although sCJD shows us different faces, perhaps there is a single facial expression. Recognising this might be helpful in diagnosis, although other diagnostic techniques (such as the established csf 14-3-3 test and the developing role of cerebral magnetic resonance imaging) will be more useful. ${ }^{4}$ However, understanding this may help us to manage patients and better explain unusual phenomena to distressed relatives. Understanding may also help us to explore further the mechanisms of this terrible disease. Perhaps the most intriguing suggestions made by the authors relate to the possible anatomic and pathological implications of their findings. 
J Neurol Neurosurg Psychiatry 2002;73:613-614

\section{Author's affiliations}

R Knight, NCJDSU, Western General Hospital,

Crewe Road, Edinburgh EH4 2XU, UK;

R.Knigh†@ed.ac.uk

\section{REFERENCES}

1 RG Will, MP Alpers, D Dormont, et al. Infectious and sporadic prion diseases. In: Prusiner SB (ed). Prion biology and diseases. New York: Cold Spring Harbour Laboratory Press, 1999:465-507.

2 Parchi P, Giese A, Capellari S, et al. Classification of sporadic Creutzfeldt-Jakob disease based on molecular and phenotypic analysis of 300 subjects. Ann Neurol 1999:46:224-33.

3 Snowden JS, Mann DMA, Neary D. Distinct neuropsychological characteristics in Creutzfeldt-Jakob disease. J Neurol Neurosurg Psychiatry 2002;73:686-694.

4 DA Collie, RI Sellar, M Zeidler, et al. MRI of Creutzfeldt-Jakob: imaging features and recommended MRl protocol. Clin Radiol 2001;56:726-39.

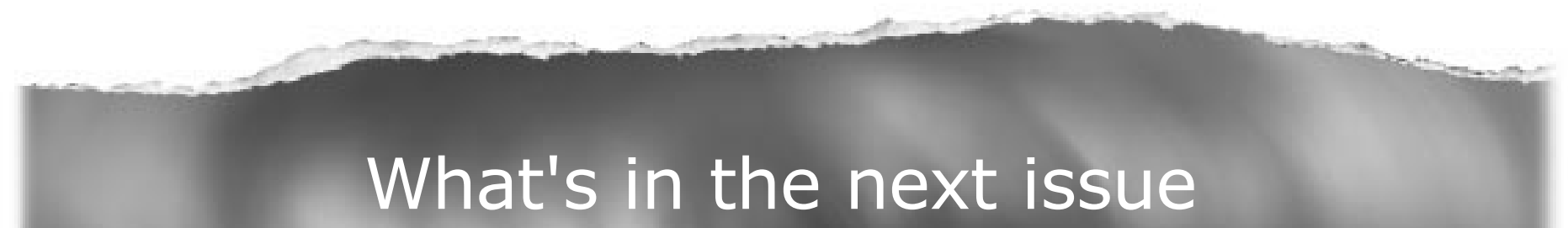

\section{Future content}

See which articles have just been accepted for publication and preview the table of contents for the next issue a month before it is published

www.jnnp.com 